\title{
Origen, desarrollo histórico y perspectivas de la Escuela Académica Profesional de Enfermería 1984-2009, en el marco de la misión de la Universidad Peruana Unión
}

\author{
Raquel Mahali CASTRO RODRÍGUEZ ${ }^{1}$, Anita Keyla FERNÁNDEZ SEGURA² y Mary Luz SOLÓRZANO APARICIO³
}

\begin{abstract}
RESUMEN
Objetivos: El objetivo de la investigación fue describir el origen, desarrollo histórico y perspectivas de la Escuela Académica Profesional de Enfermería, 1984 - 2009, en el marco de la misión de la Universidad Peruana Unión. Metodología: La investigación es de carácter histórico, los datos fueron recogidos de las actas del Consejo Universitario, actas de Consejo de Facultad, revistas, estructuras curriculares y documentos legales, que luego fueron procesados en un formato para validar la información recopilada. Además se realizó un guión de entrevista de profundidad con preguntas semiestructuradas para precisar los hechos importantes y trascendentes de la E.A.P. de Enfermería. Resultados: La Escuela Académica Profesional de enfermería, a través de los años, ha ido evolucionando de forma ascendente en el proceso de enseñanza-aprendizaje de alta calidad, debido a que cuenta con docentes altamente calificados para realizar esta labor, quienes además reciben capacitación constante para satisfacer con las expectativas de los alumnos. Por otro lado, no se dejó de impulsar la investigación en los alumnos y docentes hasta el día de hoy. En el área de proyección social y extensión universitaria, en sus inicios, se visitaron las comunidades para realizar campañas y actividades de bien social aunque no estaban dentro del sílabo. Actualmente se están incluyendo en los sílabos. Conclusión: El desarrollo de la E.A.P. de enfermería se ha regido por la filosofía, visión, misión y objetivos educacionales. A lo largo de su historia, ha colaborado con los campos misioneros, como el brazo derecho del evangelismo. La E.A.P. de enfermería responde a las exigencias de la sociedad y la iglesia actualizando los planes de estudio. Un aspecto que se debería reforzar es la evaluación más profunda del currículo de la E.A.P. con la participación de los miembros constituyentes.
\end{abstract}

Palabras claves: Historia de enfermería, Universidad Peruana Unión.

\begin{abstract}
Objective: The objective of this research was to describe the origin, historical development and prospects of Professional Academic School of Nursing, 1984 - 2009, as part of the mission of the University Peruvian Unión. Methods: The research is historical; data were collected from the records of the University Council, Faculty Council Minutes, Magazines, Curriculum Frameworks and legal documents, which were then filled in a form to validate the information gathered. Also carried out an in depth interview guide with semi-structured questions to clarify the facts essential issues of the EAP Nursing. Results: Academic School of Nursing Professional through the years has evolved from the bottom up, in the process of teaching and learning of high quality because it has highly qualified teachers for this task, they also receive ongoing training to meet expectations of students. On the other side is not stopped progress in research on students and teachers to this day. In the area of outreach and university extension in the beginning was out to communities to undertake campaigns and social welfare activities but was not in the syllabus. It has now been included in the syllabus. Conclusions: The development of E.A.P. Nursing has ruled based on the philosophy, vision, mission and educational objectives. Throughout its history the E.A.P. Nursing has collaborated with the mission fields, as the right arm of evangelism. The E.A.P. Nursing responds to the demands of society and the church by updating the curriculum. One aspect that should be strengthened further evaluation is the curriculum of the EAP with the participation of constituents.
\end{abstract}

Keywords: History of nursing, Universidad Peruana Unión.

${ }^{1}$ Licenciada en Enfermería, Universidad Peruana Unión

${ }^{2}$ Licenciada en Enfermería, Universidad Peruana Unión

${ }^{3}$ Magíster en Ciencias, con mención Enfermera Educadora, Docente de la Facultad de Ciencias de la Salud, Universidad Peruana Unión. 


\section{INTRODUCCIÓN}

Los últimos años se han caracterizado por una rápida transformación hacia nuevas formas sociales. Los cambios que afectan a las profesiones sanitarias y por tanto a la enfermería, son múltiples: Cambios internos de la profesión, estructurales de los sistemas sanitarios y cambios externos globales de la sociedad. Estos factores de cambio se pueden agrupar en económicos, sociales, culturales, políticos, demográficos, sanitarios y tecnológicos; pero, lo importante es que todos ellos están estrechamente relacionados en consecuencia, los cambios que se producen en un sector repercuten en forma directa o indirecta en el resto.

La enfermería, como profesión de servicio, está influenciada y condicionada por todos estos cambios que crean nuevos escenarios sociales y de salud, que deben afrontar las profesiones sanitarias. El profesional de enfermería no puede plantearse solamente cómo adaptarse a ellos, sino que debe entender cuáles son los valores, las necesidades y las expectativas cambiantes de la sociedad, además formarse para poder proporcionar cuidados expertos y de óptima calidad.

Asimismo, son importantes: el movimiento mundial referente al concepto de desarrollo sostenible, la diversidad cultural en la que estamos inmersos, así como el desarrollo de la cultura por la paz, entendida como conjunto de valores, actitudes y comportamientos que reflejan el respeto a la vida, al ser humano y a su dignidad y que pone en primer plano los derechos humanos, el rechazo a la violencia en todas sus formas y la adhesión a los principios de libertad, justicia, solidaridad y tolerancia (Agencia Nacional de Evaluación de la Calidad y Acreditación, 2008).

El enfermero adventista, en la práctica de su ejercicio profesional considera que el individuo fue creado con cuatro dimensiones: física, mental, social y espiritual, constituyéndose en un ser holístico. Así, mediante actividades de enfermería dirigidas a satisfacer sus necesidades básicas, logra cumplir con su filosofía cristiana, la cual es colaborar en la restauración de la imagen de Dios en el hombre (Curo, 1990).

El objetivo del presente trabajo de investigación es describir el origen, desarrollo histórico y perspectivas de la Escuela Académica Profesional de Enfermería, 1984 -2009, en el marco de la misión de la Universidad Peruana Unión.

Se desea que el alumno logre aprender de nuestra existencia como pensadores y hacedores de los cuidados de enfermería, en relación a un todo y a sus múltiples e interesantes relaciones. En este sentido tiene una gran impor- tancia profundizar y transmitir a los alumnos el interés por la investigación histórica con el fin de rescatar el pasado y crear nuevas formas de educación para el futuro.

\section{MATERIAL Y MÉTODOS}

El estudio es histórico y descriptivo, trata de la experiencia pasada, describiéndose lo que era. Esto representa una búsqueda crítica de la verdad sustentada en acontecimientos pasados. Los investigadores han dependido de fuentes primarias y secundarias, las cuales proveen la información, previo examen cuidadoso de los mismos con el fin de determinar su confiabilidad. Además, se ha verificado la autenticidad de un documento y se ha determinado el significado y la validez de los datos que contienen los documentos que se consideraban auténticos (Grajales, 1996).

La Universidad Peruana Unión cuenta con 5 facultades: (1) Facultad de Ciencias Empresariales, (2) Facultad de Ciencias humanas y Educación, (3) Facultad de Ingeniería y Arquitectura, (4) Facultad de Teología, y la (5) Facultad de Ciencias de la Salud. Cada facultad cuenta con escuelas profesionales. El estudio se realizó en la Escuela Académica Profesional de Enfermería.

En la recolección de datos se han utilizado los siguientes medios:

Guía de entrevistas: Dirigida a los que estuvieron el cargo de la decanatura. Las entrevistas se han grabado y luego fueron procesadas. Se usó un guión de entrevista de profundidad con 14 preguntas semiestructuradas.

Guía de análisis de documentos: Se ha utilizado un formato para el llenado de la información sobre programas de estudios, datos de profesores y personal de la Escuela de Enfermería, laboratorios, biblioteca, normas académicas y reglamentos, infraestructura, ingresantes, egresados, investigación, proyección social, extensión universitaria, desarrollo espiritual, servicio al alumno y convenios, todo obtenido de los libros de Actas del Consejo de Facultad de Ciencias de la Salud y las Actas del Consejo Universitario.

Asimismo, se han revisado los documentos académicos, tales como estructura curricular de la facultad, plan de estudios, sílabos, revista universitaria "El Eco", y otras publicaciones encontradas en la biblioteca, Centro White y hemeroteca. Se revisaron los documentos legales tales como, los reglamentos académicos y laborales, en cuanto a fecha de promulgación y fechas de modificación.

Antes de iniciar el estudio, se solicitó el permiso al director del Centro de Investigación de la Universidad 
Peruana Unión para realizar la entrevista dirigida al personal administrativo y docente que laboró en la $\mathrm{Fa}$ cultad de Ciencias de la Salud desde el año 1984 hasta el 2009 .

También se solicitó permiso al Secretario General para poder revisar las Actas del Consejo Universitario de los años 1984 hasta el 2009. Y del mismo modo, también se solicitó permiso a la Directora de Escuela y Secretario de la Facultad de Ciencias de la Salud para acceder a los archivos, estructuras curriculares y actas del consejo de Facultad de Ciencias de la Salud desde 1984 hasta el 2009.

Una vez recolectados los datos, se clasificaron según los objetivos, en relación a los criterios establecidos por la Asociación de Acreditación Adventista. Por otro lado, algunos de los datos fueron llenados en Excel para transformarlos en información a través de la presentación en cuadros y gráficos. Las entrevistas fueron tomadas de forma oral y escrita, debido a que algunos ex docentes no se encuentran dentro del país.

\section{CONCLUSIONES}

El desarrollo de la E.A.P. de Enfermería se ha regido en base a la filosofía, visión, misión y objetivos educacionales.
- A lo largo de su historia la E.A.P. de Enfermería ha colaborado con los campos misioneros de la IASD, como el brazo derecho del evangelismo.

- Se observa un incremento en los cuadros docentes que responden a la demanda de alumnos que se incrementan cada año.

- Asimismo, han surgido normas y reglamentos que rigen las actividades de los diferentes órganos internos y comisiones. Algunos como el Manual de Organización de Funciones necesita ser actualizado.

- $\quad$ La E.A.P. de Enfermería responde a las exigencias de la sociedad y la Iglesia Adventista del Séptimo Día (IASD) , actualizando los planes de estudio. Un aspecto que se debería reforzar es la evaluación más profunda de su currículo con la participación de los miembros constituyentes.

- La E.A.P. de Enfermería ha considerado la contratación de docentes con el perfil propuesto por la Iglesia Adventista del Séptimo Día.

- Las investigaciones realizadas por los alumnos y docentes y las capacitaciones se han incrementado.

- La participación de los alumnos cada vez es más creciente en diversas actividades académicas, sociales y de proyección social.

- En los últimos años el área de Bienestar Universitario se ha reforzado, en mejorar significativamente la biblioteca.

\section{REFERENCIAS BIBLIOGRÁFICAS}

1. Acero Tolosa, J. R. (2010). Elementos Culturales que influyen en el cuidado de enfermería de la poblacion Santandereana en el año de 2007 - 2009. Santander, Colombia: Universidad Cooperativa de Colombia.

2. Agencia Nacional de Evaluación de la Calidad y Acreditación. (2008). Libro Blanco Título de Grado de Enfermería. Zaragoza: Impresa.

3. Alarcon, N., \& Mendéz, R. (2000). La calidad y el contexto actual de la educación superior. Chile: Universidad Austral de Chile.

4. ASPEFEEN. (4 de julio de 2005). Antecedentes de la formación de enfermeras en el país. Recuperado el 11 de febrero de 2011, de http:// www.aspefeen.org.pe/estadisticas/antecedentes. php.
5. Curo Vargas, V. (1990). Orígenes de la Historia de Enfermería Adventista en el Perú. Lima: UPeU.

6. Facultad Ciencias de la Salud. (2000, 2005, 2009). Estructura Curricular. Lima: Universidad Peruana Unión.

7. Facultad de Ciencias de la Salud. (1996-2008). Actas del Concejo de Facultad. Lima: Union.

8. Universidad Peruana Union. (1988). Acta del Consejo Universitario. Lima: Unión.

9. Universidad Peruana Unión. (1984-2009). Facultad de Ciencias de la Salud. El Eco

10. UPeU. (2008). XXV Aniversario - Bodas de Plata. El Eco, 40-42. 